\title{
Analysis of a Spatial Modulation System over Time-varying Rician Fading Channel with a CSI Detector
}

\author{
Yanni Zhou, Florin Hutu and Guillaume Villemaud \\ Univ Lyon, INSA Lyon, Inria, CITI, F-69621, Villeurbanne, France
}

\begin{abstract}
Spatial Modulation (SM) is proposed in the literature as a new Multiple-Input Multiple-Output (MIMO) transmission scheme. The advantage of the SM consists in using only one RF (Radio Frequency) chain in the transmitting end which helps to increase the energy efficiency and the performance of the transmission. The related work in the literature is mostly based on the study of the SM transmission under the simple channel models. Moreover, it is assumed that the perfect Channel State Information (CSI) is known by the receiving end. This article discusses a CSI detector for the channel estimation and analyzes the performance of the proposed system over the time-varying Rician fading channel. The ADS-Matlab co-simulation results demonstrate that the bit error rate (BER) can be kept at an acceptable level with a realistic CSI detector, and in this case the system offers better performance with a small Rician factor.

Index Terms - Spatial Modulation, Rician channels, Channel Estimation, Channel State Information.
\end{abstract}

\section{INTRODUCTION}

MIMO technology plays a significant role in wireless communications. However, when multiple antennas transmit data simultaneously, high inter-channel interference is generated, which leads to a decrease in performance. It is well known that in the case of MIMO systems, the system's complexity increases with the number of antennas since each antenna is associated with an RF chain, which is increasing the total energy consumption of the transceiver. In order to overcome this issue, one solution is to use an unique RF transmission chain together with an RF switch in order to convey each emitted symbol to a particular antenna. This method is called Spatial Modulation and was proposed for the first time in [1].

SM uses the active state of antennas as a support for the transmission of information, which effectively simplifies the problems of inter-channel interference and spectrum congestion in the traditional MIMO scheme. It was explained in [2] that, compared to the V-BLAST (Vertical Bell Laboratories Layered Space-Time) system, the SM receiver does not require a continuous interference cancellation step in order to handle inter-channel interference, and has low system complexity. Moreover, the use of a unique RF link greatly reduces the cost of a SM system. SM can work normally when the number of receiving antennas is smaller than the number of transmitting antennas, which avoids the limitation of the number of receiving antennas compared to other MIMO technologies.
In the literature, [3] highlights the benefits of SM and the potential in 5G. Moreover, the design guidelines are discussed in [4]. The related techniques of SM, such as Space Shift Keying (SSK) [5], Space-Time Shift Keying (STSK) [6] and Generalized Spatial Modulation (GSM) [7] have also been proposed. Additionally, various lowcomplexity or high-accuracy detectors are also proposed. In [8], the authors present the performance analysis of a SM scheme in the case of a ML (Maximum Likelihood) detector (i. e. optimal detector). However, in their study, the channel model is a basic Additive White Gaussian Noise (AWGN) one.

Furthermore, the performance analysis over the more complex channel models has been presented in [9] and [10]. However, most of the previous studies assume that perfect CSI is available at the receiver, which may be difficult to implement in real time. The difficulty is even greater in scenarios with mobility, where there is high relative speed between the emitter and the receiver. Hence, this assumption is unrealistic. Furthermore, several authors [11], [12] have studied the effects of the channel estimation errors on the SM performances. In [13], it analyzes the performance of SM in the presence of channel estimation errors by introducing different variances of the estimation errors. However, to the best of the author's knowledge, studies on the design of the CSI detector are still lacking.

In this paper, we propose a SM system with a CSI detector. It is no longer assumed that a perfect CSI can be obtained at the receiving end. The CSI is considered to be degraded by the AWGN noise. In order to study the performance of the proposed system, Advanced Design System (ADS) - Matlab system co-simulations will be performed. More precisely, the performance of the SM over the timevarying Rician fading channel will be analysed.

The remainder of this paper is structured as follows. In Section II, the system and channel models will be discussed, and the channel model together with the channel estimation strategy will be pointed out. The ADS-Matlab co-simulation results are presented in Section III. Section IV summarizes the results of this work and gives the future directions. 


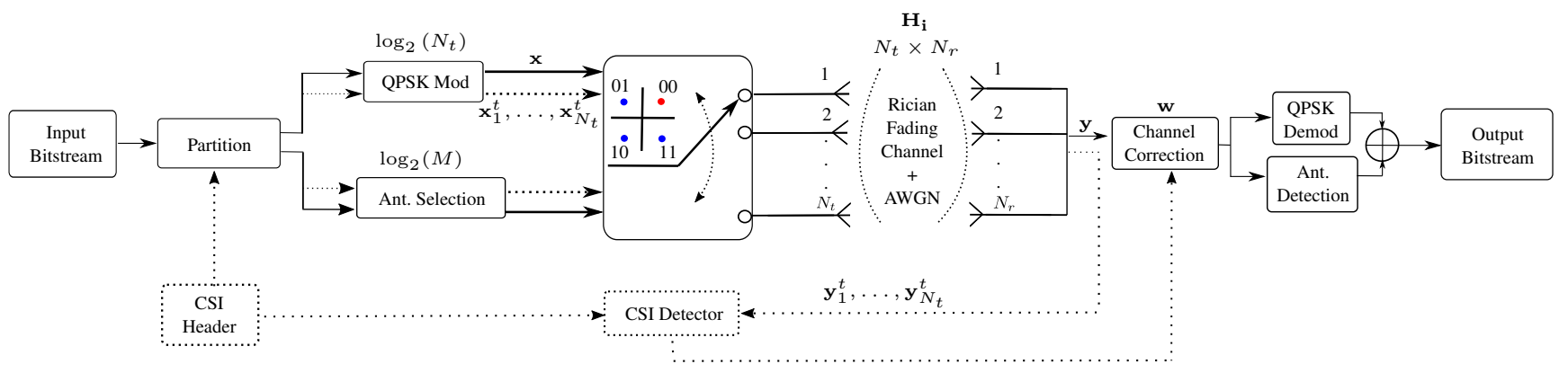

Fig. 1. $N_{t} \times N_{r}$ SM system model with a CSI Detector

\section{System Model}

A SM system model over the time-varying Rician fading channel with a CSI detector is presented in Fig.1. Here $N_{t}$ is the number of emitting antennas and $N_{r}$ is the number of receiving antennas. The SM architecture can be divided into three main parts:

1) Transmitting end: Here, the input bitstream is divided into two parts. One part is used for the antenna selection and the other one is used in order to form the symbols. As a header, the other $N_{t}$ sets of data are sent for the CSI estimation.

2) Channel: A $N_{t} \times N_{r}$ channel model is built. The activated antenna transmits the modulated symbols through a time-varying Rician fading channel. Besides, an AWGN noise is also considered.

3) Receiving end: Here, the CSI from the detector is used for the antenna detection. Moreover, each received symbol is demodulated. The resulting bits from the antenna detection are concatenated with the ones from the demodulation step, thus forming the output bitstream.

\section{A. SM modulator}

The input bit stream is divided into $N$ groups. Each group has $\log _{2}\left(N_{t}\right)+\log _{2}(M)$ bits, where $M$ is the number of the distinct symbols that the transmitter can send. The transmitter activates the corresponding antenna according to the first $\log _{2}\left(N_{t}\right)$ bits of the input bitstream. The rest $\log _{2}(M)$ bits are used in order to form the M-ary signal constellation. Besides, in the SM system, there is a limit on the number of the transmitting antennas, which is required to be $2^{n}$, so at least 2 transmitting antennas are required to ensure the normal operation of the system.

This paper deals with the particular case of the quadrature phase-shift keying (QPSK) modulation, consequently, $\log _{2}(M)=2$. A $4 \times 4$ SM mapping table is shown in TABLE I.

\section{B. Channel Model}

As stated, the symbols are transmitted over a Rician fading channel $\mathbf{H}$, where $\mathbf{H} \in \mathbb{C}^{N_{t} \times N_{r}}$. Moreover, an AWGN noise $\eta$, is considered.
TABLE I

$4 \times 4$ Spatial Modulation System Mapping TABLE

\begin{tabular}{c|cc}
\hline \multicolumn{3}{c}{$\mathrm{Nt}=4, \mathrm{M}=4$} \\
\hline Input Bits & Antenna & QPSK Symbol \\
\hline 0000 & $T_{x 1}$ & $1+\jmath$ \\
0001 & $T_{x 1}$ & $-1+\jmath$ \\
0010 & $T_{x 1}$ & $1-\jmath$ \\
0011 & $T_{x 1}$ & $-1-\jmath$ \\
0100 & $T_{x 2}$ & $1+\jmath$ \\
0101 & $T_{x 2}$ & $-1+\jmath$ \\
0110 & $T_{x 2}$ & $1-\jmath$ \\
0111 & $T_{x 2}$ & $-1-\jmath$ \\
1000 & $T_{x 3}$ & $1+\jmath$ \\
1001 & $T_{x 3}$ & $-1+\jmath$ \\
1010 & $T_{x 3}$ & $1-\jmath$ \\
1011 & $T_{x 3}$ & $-1-\jmath$ \\
1100 & $T_{x 4}$ & $1+\jmath$ \\
1101 & $T_{x 4}$ & $-1+\jmath$ \\
1110 & $T_{x 4}$ & $1-\jmath$ \\
1111 & $T_{x 4}$ & $-1-\jmath$ \\
\hline
\end{tabular}

The Rician fading channel model $\mathbf{H}$ takes into account the presence of the line of sight (LOS) path and the Rice distribution is described by the Rician factor $K$. The factor $K$ is defined as the ratio between the main signal power (usually the LOS path signal) and the sum of the powers of the remaining multipaths. In this particular case, $\mathbf{H}$ has the following structure:

$$
\mathbf{H}=\sqrt{\frac{K}{K+1}} \cdot \mathbf{A}+\sqrt{\frac{1}{K+1}} \cdot \mathbf{V}
$$

where $A$ is a deterministic matrix and $V$ is a stochastic one.

We assume that the channel parameters change every $\tau_{H}$ seconds, and the output time step is $T_{b i t}\left(\tau_{H} \gg T_{b i t}\right)$. More precisely, the same $\mathbf{H}$ matrix function is used for a set of $\tau_{H} / T_{b i t}$ symbols.

The relation between the output signal $\mathrm{y}$ and the input one $\mathbf{x}$ can be written as follows:

$$
\begin{gathered}
\mathbf{y}=\mathbf{H}_{\mathbf{i}} \cdot \mathbf{x}+\boldsymbol{\eta}, \quad i \in\left[1, \ldots, N_{H}\right] \\
N_{H}=\frac{N \cdot\left[\log _{2}\left(N_{t}\right)+\log _{2}(M)\right] \cdot T_{b i t}}{\tau_{H}}
\end{gathered}
$$


where $\mathbf{H}_{\mathbf{i}}$ is a particular realization of $\mathbf{H}, N_{H}$ is the maximum number of $\mathbf{H}$ matrix.

\section{CSI detector and demodulator}

Since the transmission information bits are respectively mapped in the antenna number and the modulation symbol, it is necessary to detect both the emitting antenna and the transmission symbol. In the detection process of the receiving end, if error occurs in the process of the antenna number detection, the estimated modulation symbol is meaningless. Therefore, the detection of the Tx antenna's number directly impacts the performance of the system, so the receiving end of the SM system needs to be mastered with more complete CSI to better detect the antenna number.

The principle of the CSI detector is based on the LeastSquares (LS) algorithm [14]. The detector calculates the channel matrix elements based on the estimated sampling co-variance matrix. More precisely, we establish $N_{t}$ sets of the incoming data. Each set is a repeating loop of $\log _{2}\left(N_{t}\right)+\log _{2}(M)$ bits. The first $\log _{2}\left(N_{t}\right)$ bits are fixed and known by the receiver in order to analyze the corresponding channel state. LS algorithm is applied for the CSI detection depending on calculating the linear relationship between the input and the output. In this situation, each set of the incoming data can get $N_{r}$ channel states, the total $N_{t}$ sets can get the real-time channel matrix $H$, where $\mathbf{H} \in \mathbb{C}^{N_{r} \times N_{t}}$.

The current CSI is used for the correction at the receiving end. The correction matrix element $\mathbf{w}_{\mathbf{i j}}$ is as follows:

$$
\mathbf{w}_{\mathbf{i j}}=\frac{1}{N^{2}} \cdot\left[\mathbf{x}_{\mathbf{i}}^{\mathbf{t}} \cdot \mathbf{x}_{\mathbf{i}}^{\mathbf{t}^{H}}\right]^{-1} \cdot \overline{\mathbf{y}_{\mathbf{i j}}^{\mathbf{t}}} \cdot \mathbf{x}_{\mathbf{i}}^{\mathbf{t}^{T}}
$$

where $\mathbf{x}_{\mathbf{i}}^{\mathbf{t}}$ is the modulated symbols sent by the $i^{\text {th }}$ transmitting antenna, and $\mathbf{y}_{\mathbf{i j}}^{\mathbf{t}}$ is the symbols received by $j^{\text {th }}$ receiving antenna which is transmitted by the $i^{\text {th }}$ transmitting antenna. The operator $[\cdot]^{H}$ denotes the Hermitian transpose and $\overline{[\cdot]}$ denotes the complex conjugate.

Because of using the QPSK modulation, in order to detect the transmitting antenna, only four possible symbols exist. The average value of the corrected demodulated signal will be compared to the four possible symbols to find the minimum difference. In other words, the most likely transmitting antenna is the antenna giving the smallest difference between the received symbol and one of the four QPSK symbols.

Finally, the binary values corresponding to the received QPSK symbol are extracted. These values are concatenated with the binary values obtained by identifying the emitting antenna.

\section{Simulation Results}

A simulator of the SM scheme (Fig. 1) was built in order to extract the performance in terms of BER variation with respect of $E b / N o$, which is the energy per bit over the noise power spectral density. We simulate the transmitting and receiving ends on Keysight's ADS and implement digital signal processing and part of the detection algorithm on Matlab. The simulation parameters are gathered in TABLE II:

TABLE II

SYSTEM PARAMETERS

\begin{tabular}{cc}
\hline \hline Parameter & Value \\
\hline Carrier frequency & $2.4 \mathrm{GHz}$ \\
Signal Power & $10 \mathrm{dBm}$ \\
Modulation type & QPSK \\
Data rate & $10 \mathrm{Mbit} / \mathrm{s}$ \\
Total number of input bits & $10^{6}$ \\
Total number of CSI test bits & $10^{5}$ \\
\hline
\end{tabular}

From Fig. 2, one can remark that the increase in the number of emitting antennas will increase the BER. More precisely, the BER performance of the $2 \times 2 \mathrm{SM}$ system is better than that of the $4 \times 4$ SM system. This is because there are only two possible transmitting antennas in the $2 \times 2 \mathrm{SM}$ system compared with four possibilities in the $4 \times 4$ SM system. In other words, the detection will be relatively easier. On the other side, the proposed channel estimation slightly increases the BER compared to the case where the perfect CSI is considered. However, it can be considered that the difference of BER performance between the system with perfect CSI and the system with imperfect estimated CSI is at acceptable level.

From Fig. 3, one can remark that the SM system over a Rician fading channel offers better performance for the smaller values of $\mathrm{K}$. This can be explained because the difference between the $N_{t} \times N_{r}$ values in the channel matrix $\mathbf{H}$ is minimal if the $K$ factor is significant, which leads to imprecision in the antenna detection. In other words, if the CSI difference between each channel is

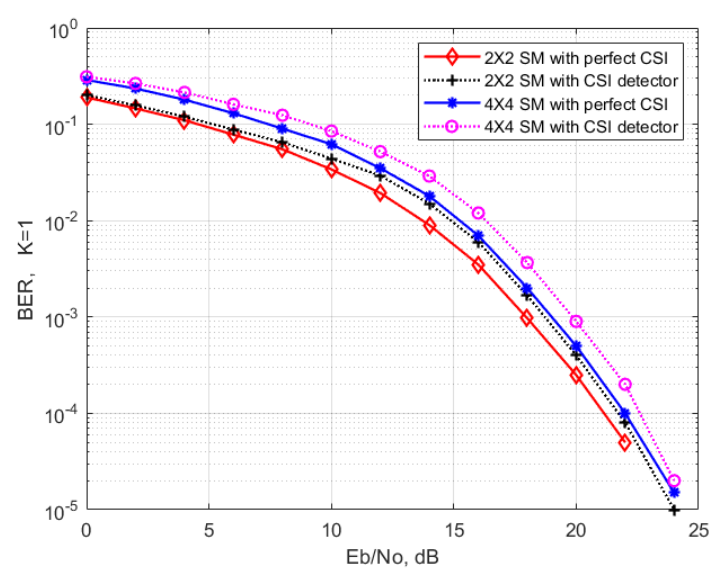

Fig. 2. BER performance of the $2 \times 2$ and $4 \times 4$ SM system with the perfect and realistic CSI detectors over time-varying Rician fading channel 


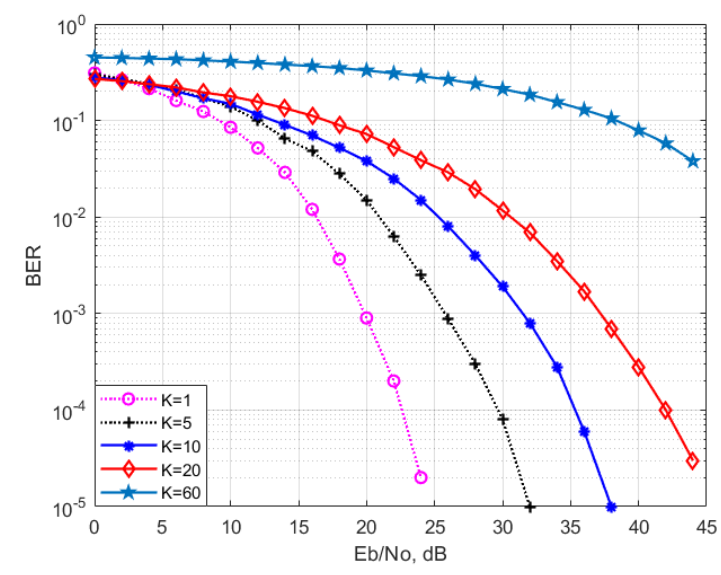

Fig. 3. BER performance of the $4 \times 4$ SM system with the realistic CSI detector and for different Rician factor $K$

assumed to be negligible, all $N_{t} \times N_{r}$ elements in the channel matrix $\mathbf{H}$ are equal, then the BER of detecting the transmitting antenna will remain at 0.5 . Hence, the more multipaths in the channel, the better we can detect the emitting antenna.

\section{CONCLUSION}

In this paper, a SM system over the time-varying Rician fading channel was presented. The modulation module as well as the channel model were introduced. In particular, a time-varying CSI detector for the channel estimation was designed instead of assuming the availability of the perfect $\mathrm{CSI}$ at the receiver. System simulation results indicate that the detector error is acceptable when the Rician Factor $\mathrm{K}$ is small. It is envisaged to improve the simulation framework by taking into account the receiver non-idealities (local oscillator's phase noise, presence of nonlinear effects, etc.) and extending the result to more complex modulations. Moreover, an experimental framework based on National Instruments' VST (Vector Signal Transceiver) platform is envisaged.

\section{REFERENCES}

[1] R. Mesleh, H. Haas, C. W. Ahn, and S. Yun, "Spatial ModulationOFDM,” pp. 288-292, 2006.

[2] M. D. Renzo, H. Haas, and P. M. Grant, "Spatial modulation for multiple-antenna wireless systems: a survey," IEEE Communications Magazine, vol. 49, no. 12, pp. 182-191, December 2011.

[3] D. T. Phan-huy et al, "Single-Carrier Spatial Modulation for the Internet of Things: Design and Performance Evaluation by Using Real Compact and Reconfigurable Antennas," IEEE Access, vol. 7, pp. 18 978-18 993, 2019.

[4] P. Yang, M. D. Renzo, Y. Xiao, S. Li, and L. Hanzo, "Design Guidelines for Spatial Modulation," IEEE Communication Surveys \& Tutorials, vol. 17, no. 1, pp. 6-26, 2014.

[5] J. Jeganathan, A. Ghrayeb, L. Szczecinski, and A. Ceron, "Space shift keying modulation for MIMO channels," IEEE Transactions on Wireless Communications, vol. 8, no. 7, pp. 3692-3703, July 2009.

[6] S. Sugiura, S. Chen, and L. Hanzo, "Coherent and differential space-time shift keying: A dispersion matrix approach," IEEE Transactions on Communications, vol. 58, no. 11, pp. 3219-3230, November 2010

[7] A. Younis, N. Serafimovski, R. Mesleh, and H. Haas, "Generalised spatial modulation," in 2010 Conference Record of the Forty Fourth Asilomar Conference on Signals, Systems and Computers, Nov 2010, pp. 1498-1502.

[8] J. Jeganathan, A. Ghrayeb, and L. Szczecinski, "Spatial Modulation : Optimal Detection and Performance Analysis," IEEE Communications Letters, vol. 12, no. 8, pp. 545-547, 2008.

[9] M. D. Renzo and H. Haas, "On the Performance of Space Shift Keying MIMO Systems Over Correlated Rician Fading Channels," in IEEE International ITG Workshop on Smart Antennas, 2010.

[10] M. M. Alwakeel, "Quadrature Spatial Modulation Performance Analysis over Rician Fading Channels," Journal of Communications, vol. 11, no. 3, pp. 249-254, 2016.

[11] A. Afana, N. Abu-ali, and S. Ikki, "On the Joint Impact of Hardware and Channel Imperfections on Cognitive Spatial Modulation MIMO Systems : Cramer - Rao Bound Approach," IEEE Systems Journal, vol. 13 , no. 2, pp. 1250-1261, 2018.

[12] S. Sugiura and L. Hanzo, "Effects of Channel Estimation on Spatial Modulation," IEEE Signal Processing Letters, vol. 19, no. 12, pp. 805-808, 2012.

[13] E. Basar, Ü. Aygölu, E. Panayirci, and H. V. Poor, "Performance of Spatial Modulation in the Presence of Channel Estimation Errors," IEEE Communications Letters, vol. 16, no. 2, pp. 176-179, 2012.

[14] D. Neumann, M. Joham, and W. Utschick, "Channel estimation in massive MIMO systems," 2015. [Online]. Available: http://arxiv.org/abs/1503.08691 


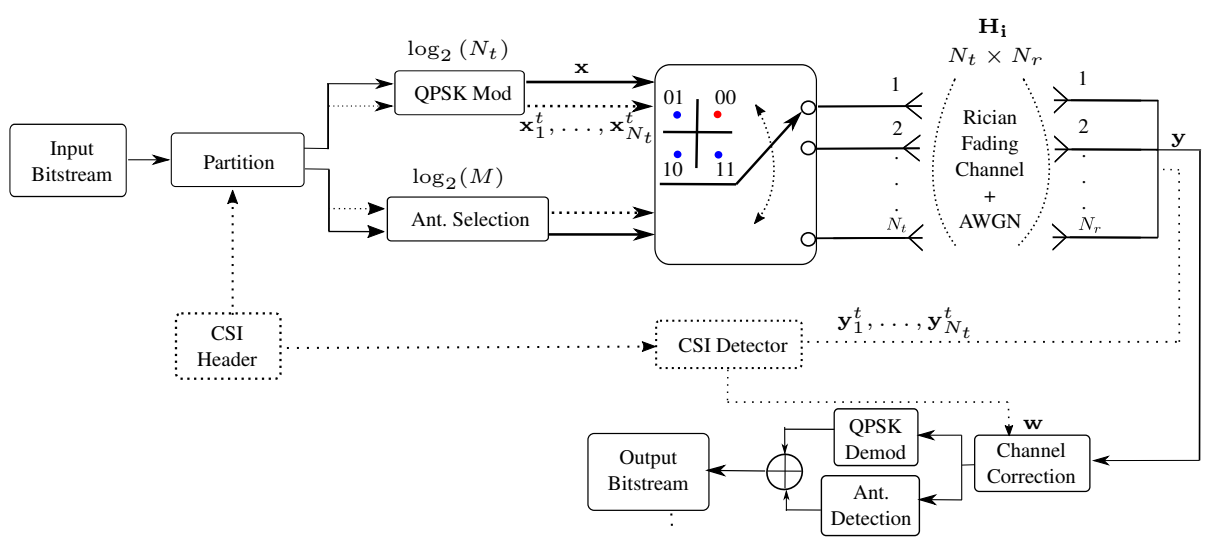

Fig. 4. $N_{t} \times N_{r}$ SM system model with a CSI Detector 